\title{
GAUSSIAN PROCESS DYNAMIC MODELING AND BACKSTEPPING SLIDING MODE CONTROL FOR MAGNETIC LEVITATION SYSTEM OF MAGLEV TRAIN ${ }^{1}$
}

\author{
YOUGANG SUN \\ Institute of Rail Transit, Tongji University, Shanghai, China, and \\ National Maglev Transportation Engineering R\&D Center, Tongji University, China \\ Sumei Wang, Yang Lu \\ National Rail Transit Electrification and Automation Engineering Technology Research Centre, \\ Hong Kong Branch, Hong Kong Polytechnic University, China \\ e-mail: may.sm.wang@polyu.edu.hk \\ JUNQI XU \\ National Maglev Transportation Engineering R\&D Center, Tongji University, China
}

The maglev trains are strongly nonlinear and open-loop unstable systems with external disturbances and parameters uncertainty. In this paper, the Gaussian process method is utilized to get the dynamic parameters, and a backstepping sliding mode controller is proposed for magnetic levitation systems (MLS) of maglev trains. That is, for a MLS of a maglev train, a nonlinear dynamic model with accurate parameters is obtained by the Gaussian process regression method, based on which a novel robust control algorithm is designed. Specifically, the MLS is divided into two sub-systems by a backstepping method. The inter virtual control inputs and the Lyapunov function are constructed in the first sub-system. For the second sub-system, the sliding mode surface is constructed to fulfil the design of the whole controller to asymptotically regulate the airgap to a desired trajectory. The stability of the proposed control method is analyzed by the Lyapunov method. Both simulation and experimental results are included to illustrate the superior performance of the presented method to cope with parameters perturbations and external disturbance.

Keywords: maglev train, Gaussian process, sliding mode control, parameter perturbations

\section{Introduction}

Maglev transportation technology has developed rapidly in the recent decades, and many maglev lines have appeared all over the world with the advantages of zero friction, stronger turning ability, faster speed potential and environment friendly, as shown in Fig. 1 (Lee et al., 2006; Yan, 2008; Thornton, 2009). Because of human's unremitting pursuit of traffic speed and comfort, maglev transportation will flourish in the near future. The magnetic levitation technology is one of the key technologies of maglev transportation. The modeling and control of the magnetic levitation system (MLS) is the premise to ensure the high-quality operation of maglev vehicles (Boldea et al., 2017).

The modeling of the MLS is relatively mature. However, values of the model parameters are not easy to obtain, and the parameters will be perturbed during vehicle operation. In addition, the model has a strong nonlinearity and is easy to be disturbed, which brings difficulties to the

\footnotetext{
${ }^{1}$ Paper presented at the 4th International Conference on Material Strength and Applied Mechanics, MSAM 2021
} 
(a)

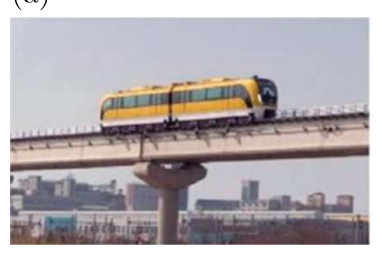

(b)

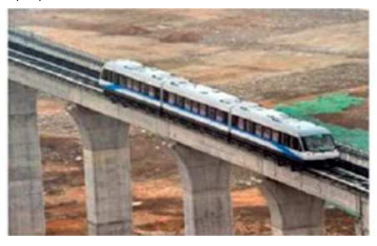

(c)

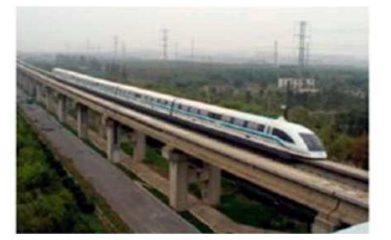

(d)

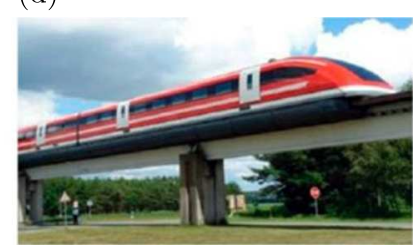

Fig. 1. Maglev transportation line all over the world

design of the controller. The traditional magnetic levitation control method is designed based on the linearization model near the equilibrium point, such as the PID controller, state feedback controller, etc. These methods can adjust the airgap, but when the system state is far from the equilibrium point, the control effect is greatly reduced, not to mention the ability of dealing with parameters change or disturbances. The airgap of an EMS maglev train is about $8.5 \mathrm{~mm}$. Therefore, the decline of levitation control performance will lead to the train colliding the rail, resulting in serious safety problems.

In the recent decades, researchers from all over the world have proposed a variety of magnetic levitation control algorithms and achieved certain results. MacLeod and Goodall (1996) combined the FDW (frequency domain weighting) method and the LQR levitation controller to improve the ability to restrain the influence from the rail irregularity. Sinha et al. (1993) and Sinha and Pechev (1999) presented a model reference adaptive controller (MRAC) with to deal with the parameters change and nonlinearity. The airgap error was optimized by utilizing an evaluation function. Sun et al. (2019) designed an adaptive Neural-Fuzzy Robust Controller with sliding mode control and a neural-fuzzy switching law, which could suppress the influence of the external interference and with smooth control inputs. Li et al. (2015) presented a levitation control law with a virtual energy harvester to remove the unexpected oscillation for a maglev vehicle and a track when the train is standing still on the track. Morales et al. (2011) combined precise linearization with output feedback control to propose a GPI controller, which was based on a fast online algebraic parameter estimation. Experimental results were included to show the effectiveness of the tracking control. Sun et al. (2019) presented a maglev train-track couping dynamic model with a flexible track and designed a feedback controller based on fuzzy logic system to remove the vehicle-rail coupling vibration. Additionally, with the rise of AI (artificial intelligence), many intelligent control algorithms such as neural network (Wu et al., 2008; Wai and Lee, 2008), genetic algorithm (Kusagawa et al., 2004), support vector machine (Liu and Rong, 2015), Monte Carlo simulation (Zhang et al., 2013) and so on, have begun to explore in the field of magnetic levitation systems. However, these control algorithms cannot strictly prove the stability of the whole system with a feedback controller theoretically. Only simulation or experiment results can be utilized to show their partial performance. Unfortunately, when the magnetic levitation system parameters perturb greatly, some rules of these algorithms should be relearned or retuned, which brings great difficulties to engineering applications. Furthermore, in the actual operation of maglev trains, some system parameters cannot be obtained accurately in advance, such as levitation mass, inductance, etc., which makes the system modeling more difficult. Therefore, an excellent magnetic levitation control algorithm needs to obtain an accurate dynamic model in advance and is able to carry out a strict stability proof.

In this paper, the nonlinear mathematical model of the MLS of a maglev train is established and analyzed firstly. The Gaussian process is used to get accurate parameter values of the model. Then, the controller is proposed directly based on the nonlinear dynamic model without any linearization. Finally, sufficient simulation and experimental results demonstrate the presented method has superior dynamic performance and robustness. 


\section{Dynamics development and analysis}

The maglev train structure and a single magnetic levitation system can be illustrated in Figs. 2 and 3 .

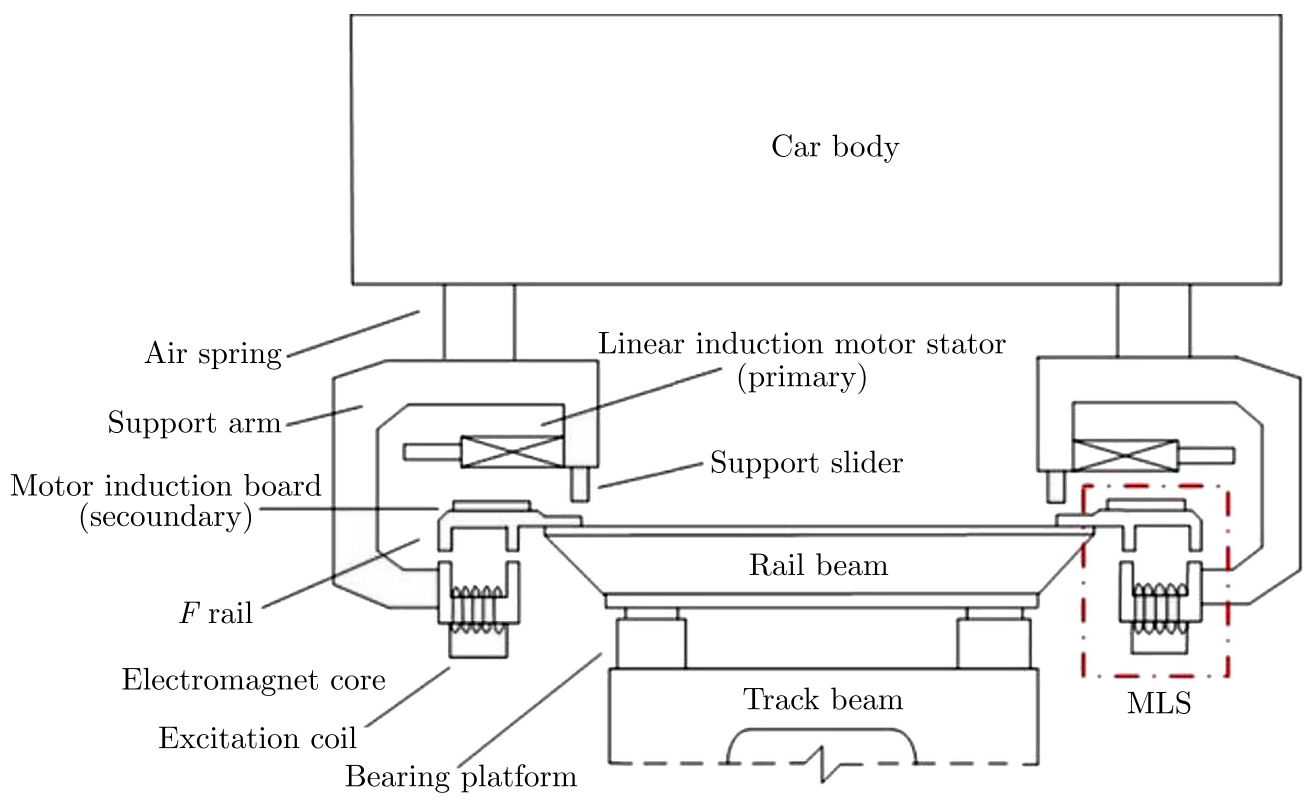

Fig. 2. Maglev train structure

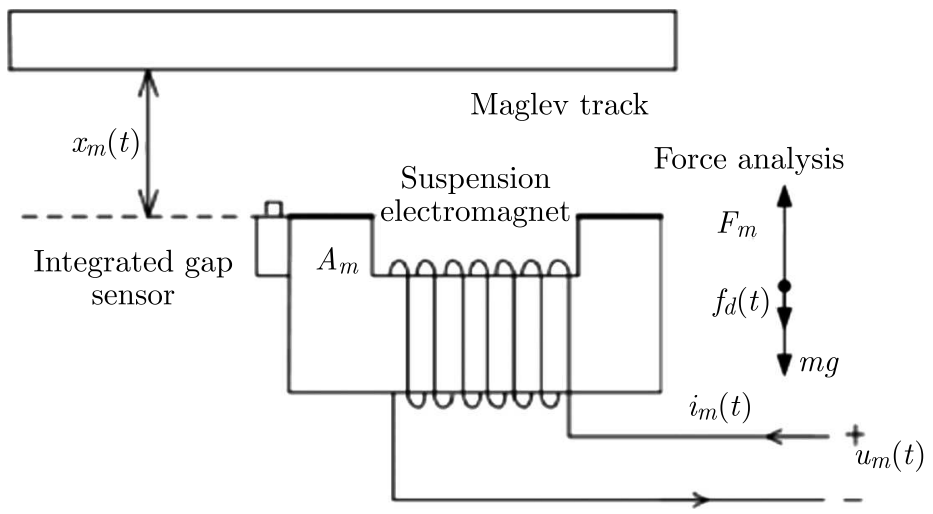

Fig. 3. Single magnetic levitation system (MLS)

Based on the Maxwell equations and the Biot-Savart theorem (Sun et al., 2020), the conventional dynamic system equations of the maglev train levitation system can be obtained as follows (Sun et al., 2019)

$$
\begin{aligned}
& m \frac{d^{2} x_{m}(t)}{d t^{2}}=-\frac{\mu_{0} A_{m} N_{m}^{2}}{4}\left(\frac{i_{m}(t)}{x_{m}(t)}\right)^{2}+m g+f_{d} \\
& \frac{d i_{m}(t)}{d t}=\frac{i_{m}(t)}{x_{m}(t)} \frac{d x_{m}(t)}{d t}+\frac{2 x_{m}(t)}{\mu_{0} N_{m}^{2} A_{m}}\left[u_{m}(t)-i_{m}(t) R_{m}\right]
\end{aligned}
$$

where $x_{m}(t)$ is the airgap between electromagnet and track beam, $A_{m}$ is the effective magnetic pole area, $\mu_{0}$ is the magnetic permeability of the air, $f_{d}$ is the external interference force. 
Convert it to a state space expression. The system status parameters selected here are $x_{1}(t)=x_{m}(t), x_{2}(t)=\dot{x}_{m}(t)$ and $x_{3}(t)=i_{m}(t)$. Then the state space expression of $(2.1)$ is

$$
\begin{aligned}
& \dot{x}_{1}(t)=x_{2}(t) \\
& \dot{x}_{2}(t)=-\frac{\mu_{0} A_{m} N_{m}^{2}}{4 m}\left(\frac{x_{3}(t)}{x_{1}(t)}\right)^{2}+g+\frac{1}{m} f_{d} \\
& \dot{x}_{3}(t)=\frac{x_{2}(t) x_{3}(t)}{x_{1}(t)}+\frac{2 x_{1}(t)}{\mu_{0} N_{m}^{2} A_{m}}\left[u_{m}(t)-x_{3}(t) R_{m}\right]
\end{aligned}
$$

Assumption 1: Maglev trains will change the mass of the levitation when passengers get on and off, assume that the main parameters of the system $m$ are perturbed, which is $m-m_{\min } \leqslant$ $\Delta m \leqslant m-m_{\max }$.

Assumption 2: External disturbance terms are observable and bounded, there is a known positive number $D$, making $f_{d} / m \leqslant D$.

Definition 1: For nonlinear systems

$$
\dot{\mathbf{x}}=\mathbf{f}(x) \quad \mathbf{x} \in R^{n}
$$

The system that linearizes at the equilibrium point is

$$
\dot{\mathbf{x}}=\mathbf{A}\left(\mathbf{x}_{0}\right)\left(\mathbf{x}-\mathbf{x}_{0}\right) \quad \mathbf{x} \in R^{n}
$$

where $\mathbf{x}_{0}$ is an isolated singularity of nonlinear system (2.3). If the real part of all the eigenvalues of $\mathbf{A}\left(\mathbf{x}_{0}\right)$ is non-zero, then $\mathbf{x}_{0}$ is the double singular point of equation (2.3).

The equivalent system of nonlinear system (2.3) is

$$
\dot{\mathbf{x}}=\mathbf{A}\left(\mathbf{x}_{0}\right)\left(\mathbf{x}-\mathbf{x}_{0}\right)+\mathbf{O}\left(\mathbf{x}-\mathbf{x}_{0}\right) \quad \mathbf{x} \in R^{n}
$$

Theorem 1 (Hartman-Grobman theorem): If $\mathbf{x}_{0}$ is a double-curve point of nonlinear system (2.2), and the conditions are met

$$
\lim _{\mathbf{x} \rightarrow \mathbf{x}_{0}}\left(\frac{\mathbf{O}\left(\mathbf{x}-\mathbf{x}_{0}\right)}{\left|\mathbf{x}-\mathbf{x}_{0}\right|}\right)=\mathbf{0}
$$

then they have the same topology in nonlinear system (2.3) at the isolated singularity $\mathbf{x}_{0}$ and linearized system at its equilibrium point (2.5).

According to Theorem 1, the linear magnetic analysis method can be used to analyze characteristics of the nonlinear MLS, and the accuracy of the analysis results can be ensured with a certain precision.

Next, solve the equilibrium point of the nonlinear MLS, that is, the system singularity, which must satisfy $\dot{\mathbf{x}}=\left(\dot{x}_{1}, \dot{x}_{2}, \dot{x}_{3}\right)^{\mathrm{T}}=\mathbf{0}$, so the system equilibrium point $\mathbf{x}_{0}=\left(x_{r e f}, \dot{x}_{r e f}, i_{r e f}\right)$, the singular point of the nonlinear system can be calculated as

$$
\mathbf{x}_{0}=\left(x_{r e f}, \dot{x}_{r e f}, i_{r e f}\right)=\left(x_{r e f}, 0, \sqrt{\kappa} x_{r e f}\right) \quad \kappa \triangleq \frac{4 m g}{\mu_{0}} N_{m}^{2} A_{m}
$$

The equilibrium point of the magnetic levitation system is the double singularity of the system. Therefore, the stability of the MLS near the equilibrium point can be studied by the linearized system. 
Through linearization operator (2.8) of the system, the Jacobian matrix of the system can be calculated

$$
\mathbf{D} f\left(\mathbf{x}_{0}\right)=\left[\begin{array}{ccc}
\frac{\partial f_{1}}{\partial y_{1}}\left(\mathbf{x}_{0}\right) & \cdots & \frac{\partial f_{1}}{\partial y_{n}}\left(\mathbf{x}_{0}\right) \\
\vdots & \ddots & \vdots \\
\frac{\partial f_{n}}{\partial y_{1}}\left(\mathbf{x}_{0}\right) & \cdots & \frac{\partial f_{n}}{\partial y_{n}}\left(\mathbf{x}_{0}\right)
\end{array}\right]
$$

In order to make the symbolic expression concise, the current coefficient $P_{i}$, the airgap coefficient $P_{x}$ and the electromagnet inductance $L_{r e f}$ at the equilibrium point are introduced, respectively

$$
P_{i}=\frac{\mu_{0} N_{m}^{2} A_{m} i_{r e f}}{2 x_{r e f}^{2}} \quad P_{x}=\frac{\mu_{0} N_{m}^{2} A_{m} i_{r e f}^{2}}{2 x_{r e f}^{3}} \quad L_{r e f}=\frac{\mu_{0} N_{m}^{2} A_{m}}{2 x_{r e f}}
$$

System (2.2) is linearized near the equilibrium point, and the coefficient matrix is got as follows

$$
\mathbf{A}_{0}=\left[\begin{array}{ccc}
0 & 1 & 0 \\
\frac{P_{x}}{m} & 0 & -\frac{P_{i}}{m} \\
0 & \frac{P_{i}}{L_{r e f}} & -\frac{R_{m}}{L_{r e f}}
\end{array}\right] \quad \quad \mathbf{A}_{1}=\left[\begin{array}{c}
0 \\
0 \\
1 \\
\frac{1}{L_{r e f}}
\end{array}\right]
$$

and the output matrix $\mathbf{C}=\left[\begin{array}{lll}1 & 0 & 0\end{array}\right]$.

Then, at the equilibrium point, magnetic levitation system (2.2) can be expressed as

$$
\dot{\mathbf{x}}=\mathbf{A}_{0}\left(\mathbf{x}_{0}\right)\left(\mathbf{x}-\mathbf{x}_{0}\right)+\mathbf{A}_{1}\left(u_{m}-u_{0}\right)+\mathbf{O}\left(\mathbf{x}-\mathbf{x}_{0}\right)^{n}
$$

Ignoring the external disturbance of the system, equation (2.11) can be obtained by the Laplace transform

$$
\left(m s^{2}-P_{x}\right) \Delta x(s)=-P_{i} \Delta i(s) \quad \Delta u(s)=\left(R_{m}+L_{r e f} s\right) \Delta i(s)-P_{i} s \Delta x(s)
$$

By combining equations (2.12), the transfer function of the MLS can be written through simple mathematical changes as follows

$$
G(s)=\frac{\Delta x(s)}{\Delta u(s)}=\frac{-P_{i}}{L_{r e f} m s^{3}+R_{m} m s^{2}+\left(P_{i}^{2}-L_{r e f} P_{x}\right) s-R_{m} P_{x}}
$$

Theorem 2 (Hurwitz stability criterion): If the characteristic equation of the system is

$$
a_{n} s^{n}+a_{n-1} s^{n-1}+\cdots+a_{1} s+a_{0}=0
$$

Then, the necessary condition for the stability of the linear system is that the coefficients of the characteristic polynomials are all positive, that is

$$
\begin{array}{llll}
a_{n}>0 & a_{n-1}>0 & \ldots & a_{0}>0
\end{array}
$$

For transfer function of the MLS (2.13), obviously, in the characteristic polynomial, it can be known from Theorem 2 that the system is unstable. 


\section{Gaussian process regression modeling}

It can be seen from the above dynamic equations that the levitation mass and the precision of electromagnet parameters have an important influence on the accuracy of the model. However, due to the change of mass and the complexity of electromagnet parameters, there is a certain gap between the mass and electromagnet parameters obtained by the equivalent method and the actual value. Therefore, there is a big gap between the general standard dynamic equations and the actual system.

The Gaussian process is a nonparametric regression method based on probability (Quiñonero-Candela et al., 2005). Given the input $X$ and output $Y$, the task of regression is to learn a mapping relationship between the input and output, and use this mapping relationship to predict the corresponding output of other inputs. Suppose that the mapping relationship between the input and output variables is $\mathbf{y}=f(\mathbf{x})$, and there are $y^{1}, \ldots, y^{n} \sim N(0, \Sigma)$. Among them, $\Sigma_{p q}=\operatorname{Cov}\left(y_{p}, y_{q}\right)=C\left(x_{p}, x_{q}\right)$ is the covariance between the outputs. Therefore, through the average value $\mu(x)$ (usually 0 ) and covariance function $C\left(x_{p}, x_{q}\right)$ one can completely describe the Gaussian process. Suppose an $N$-dimensional input vector $\mathbf{x}=\left[x_{1}, x_{2}, \ldots, x_{n}\right]$ and $N$-dimensional output $\mathbf{y}=\left[y_{1}, y_{2}, \ldots, y_{n}\right]^{\mathrm{T}}$. Given these data, given a new input $\mathbf{x}^{*}$, we hope to get the corresponding predicted distribution of the output $\mathbf{y}^{*}$. For the input $\mathbf{x}^{*}$, the prediction distribution of the corresponding output $\mathbf{y}^{*}$ is $\mathbf{y}^{*} \mid \mathbf{x}^{*},(\mathbf{x}, \mathbf{y})$, and satisfies the Gaussian distribution

$$
\left.\boldsymbol{\mu}\left(\mathrm{x}^{*}\right)=\mathbf{k}\left(\mathrm{x}^{*}\right)^{\mathrm{T}} \mathbf{K}^{-1} \mathbf{y} \quad\right] \boldsymbol{\sigma}^{2}\left(\mathrm{x}^{*}\right)=\mathbf{k}\left(\boldsymbol{x}^{*}\right)-\mathbf{k}\left(\mathrm{x}^{*}\right)^{\mathrm{T}} \mathbf{K}^{-1} \mathbf{k}\left(\mathrm{x}^{*}\right)+\boldsymbol{v}_{0}
$$

where $\mathbf{k}\left(\mathbf{x}^{*}\right)=\left[C\left(x^{1}, \mathbf{x}^{*}\right), \ldots, C\left(x^{N}, \mathbf{x}^{*}\right)\right]^{\mathrm{T}}$ is the covariance vector between the training data and the test data, and $\mathbf{k}\left(\mathbf{x}^{*}\right)=C\left(\mathbf{x}^{*}, \mathbf{x}^{*}\right)$ is the covariance of the test data $\mathbf{x}^{*}$ and itself. In this way, the output corresponding to the new input can be predicted by the existing training data. The airgap, change speed and acceleration of the airgap are selected as the input variables of the regression model, and the output variables are the output of the controller. After the input and output variables are obtained, the Gaussian process regression method can be used to estimate the dynamic model. This paper uses the experimental data for training and testing, so as to get more accurate electromagnet parameters and mass parameters information.

\section{Controller design and analysis}

The system errors of the first-level subsystem can be defined as follows

$$
e_{1}=x_{1}-x_{d} \quad \dot{e}_{1}=\dot{x}_{1}-\dot{x}_{d}=x_{2}-\dot{x}_{d}
$$

where $x_{d}$ denotes the reference trajectory, $e_{1}$ represents the error of the first-level subsystem.

According to the backstepping method, the virtual control input of the first-level subsystem can be defined as $u_{1, d}(t)$, then we can get

$$
\dot{e}_{1}=u_{1, d}-\dot{x}_{d}+e_{2}
$$

where $e_{2}(t)$ denotes the auxiliary signal error

$$
e_{2}(t)=x_{2}(t)-u_{1, d}(t)
$$

According to the system error, $u_{1, d}(t)$ can be designed as follows

$$
u_{1, d}(t)=\dot{x}_{d}-c_{1} e_{1}
$$

where $c_{1} \in R^{+}$denotes the control factor. 
Substituting (4.4) into (4.2), we can obtain

$$
\dot{e}_{1}=e_{2}-c_{1} e_{1}
$$

For (4.5), it is easy to see that if $e_{2}(t) \rightarrow 0$, then $e_{1}(t) \rightarrow 0$. For this purpose, we can select the Lyapunov candidate function as follows

$$
V_{1}=\frac{1}{2} e_{1}^{2}
$$

It can be obtained by deriving from both sides of (4.6) as follows

$$
\dot{V}_{1}=e_{1} \dot{e}_{1}=e_{1} e_{2}-c_{1} e_{1}^{2}
$$

In the design of the second-level subsystem, we should make $e_{2}(t) \rightarrow 0$ as much as possible.

Taking time derivative on both sides of (4.3), we can obtain

$$
\dot{e}_{2}(t)=\dot{x}_{2}(t)-\dot{u}_{1, d}(t)=g+\frac{1}{m} f_{d}-\frac{K}{m x_{1}^{2}} i_{m}^{2}-\ddot{x}_{d}+c_{1} \dot{e}_{1}
$$

where $K=\left(\mu_{0} A_{m} N_{m}^{2}\right) / 4 ; i_{m}$ represents the control current.

According to the sliding mode control (SMC) theory, the sliding surface is presented as below

$$
s=e_{2}=x_{2}-\dot{x}_{d}+c_{1} e_{1}
$$

For the next-level subsystem, a novel Lyapunov candidate function is constructed as follows

$$
V_{2}=V_{1}+\frac{1}{2} e_{2}^{2}=\frac{1}{2} e_{1}^{2}+\frac{1}{2} e_{2}^{2}
$$

Taking time derivative on both sides of (4.10), we can obtain

$$
\dot{V}_{2}=\dot{V}_{1}+e_{2} \dot{e}_{2}=e_{1} e_{2}-c_{1} e_{1}^{2}+e_{2}\left(g+\frac{1}{m} f_{d}-\frac{K}{m x_{1}^{2}} i_{m}^{2}-\ddot{x}_{d}+c_{1} \dot{e}_{1}\right)
$$

To guarantee $\dot{V}_{2} \leqslant 0$, the proposed controller is designed as follows

$$
i_{m}^{2}=\frac{m x_{1}^{2}}{K}\left[g-\ddot{x}_{d}+e_{1}+c_{1} \dot{e}_{1}+c_{2} e_{2}+\eta \operatorname{sgn}\left(e_{2}\right)\right]
$$

where $c_{2}>0, \eta \geqslant D$ and

$$
\dot{V}_{2}=\frac{f_{d}}{m} e_{2}-\eta e_{2} \operatorname{sgn}\left(e_{2}\right)-c_{1} e_{1}^{2}-c_{2} e_{2}^{2} \leqslant-c_{1} e_{1}^{2}-c_{2} e_{2}^{2}-(\eta-D)\left|e_{2}\right| \leqslant-c_{1} e_{1}^{2}-c_{2} e_{2}^{2}
$$

where $c_{2}>0, \eta \geqslant D$.

The proof of stability is as follows

$$
\dot{V}_{2} \leqslant-c_{1} e_{1}^{2}-c_{2} e_{2}^{2} \leqslant 0
$$

Because if $\dot{V}=-\eta V$, that is $V^{-1} d V=-\eta d t$, the integral is $\int_{0}^{t} V^{-1} d V=-\int_{0}^{t} d t$.

Then, $\left.\ln V\right|_{0} ^{t}=-\eta t$.

Thus the form of exponential convergence $V(t)=V(0) e^{-\eta t}$ is obtained.

Decompose $V_{2}=\left(e_{1}^{2}+e_{2}^{2}\right) / 2$, and easy to get $e_{1}$ and $e_{2}$ are exponentially convergent, when $t \rightarrow \infty, e_{1} \rightarrow 0$ and $e_{2} \rightarrow 0$. That is $\lim _{t \rightarrow \infty} x_{1}=x_{d}$, and since $e_{2}=x_{2}+c_{1} e_{1}-\dot{x}_{d}$, then $\lim _{t \rightarrow \infty} x_{2}=\dot{x}_{d}$. 
In order to avoid or weaken the chattering phenomenon in SMC, we utilize a function sat $(\cdot)$ with the boundary layer to replace sgn $(\cdot)$, which can guarantee that the proposed controller (4.12) can be activated outside the boundary layer, and within the boundary layer, the continuous controller is activated

$$
\operatorname{sat}(S)=\left\{\begin{array}{lll}
\frac{S}{\phi_{0}} & \text { for } & |S| \leqslant \phi_{0} \\
\operatorname{sgn}(S) & \text { for } & |S|>\phi_{0}
\end{array}\right.
$$

Finally, the proposed controller is designed as follows

$$
i_{m}^{2}=\frac{m x_{1}^{2}}{K}\left[g-\ddot{x}_{d}+c_{1} \dot{e}_{1}+c_{2} e_{2}+\eta \operatorname{sat}\left(e_{2}\right)+e_{1}\right]
$$

\section{Numerical simulation}

The parameters of the MLS can be obtained through the Gaussian process method shown in Table 1.

Table 1. Parameter values of the MLS

\begin{tabular}{|l|c|l|c|}
\hline \multicolumn{1}{|c|}{ Parameters } & Value & \multicolumn{1}{c|}{ Parameters } & Value \\
\hline \hline Mass $[\mathrm{m} / \mathrm{kg}]$ & 750 & $\mu_{0}\left[\mathrm{Hm}^{-1}\right]$ & $4 \pi \cdot 10^{-7}$ \\
\hline Number of coil turns $N_{m}$ & 450 & Reference airgap $x_{r e f}[\mathrm{~m}]$ & 0.008 \\
\hline Area of coil $A_{m}\left[\mathrm{~m}^{2}\right]$ & 0.024 & Initial airgap $x_{0}[\mathrm{~m}]$ & 0.016 \\
\hline Coil resistance $R_{m}[\Omega]$ & 1.2 & & \\
\hline
\end{tabular}

The control parameters $c_{1}, c_{2}$ and $\eta$ of the proposed controller are tuned sufficiently to obtain the best performance, which yields the following values: $c_{1}=6, c_{2}=10.5, \eta=1.5$. In order to verify the superiority of the proposed controller, we compare the proposed controller with the PID and fuzzy PID controller (Carvajal et al., 2020). The stability region of the PID controller for the MLS of maglev trains is very small. The trial-and-error method is used to determine the best control parameters of the PID, which are: $P=70000, I=2000, D=9000$. Subsequently, we verify the performance of the proposed controller, PID controller and fuzzy PID under the following three cases:

- Case 1. Static levitation without disturbances and parameter perturbations.

- Case 2. External disturbance, shown in Fig. 7, applied to the system.

- Case 3. Parameter perturbations included. For the maglev train, the mass always changes when passengers pick-up and drop-off. So a change in the levitated mass $m$ is mainly considered.

Case 1. Track of the desired position without external disturbances

The simulation results of the proposed controller, PID and fuzzy PID controller are illustrated in Figs. 4 and 5.

It can be seen from Figs. 4 and 5 that under the action of the PID controller, there is a static error of $0.25 \mathrm{~mm}$ and $0.18 \mathrm{~mm}$ for the fuzzy PID. The maximum control circuit of the PID and fuzzy PID reached $200 \mathrm{~A}$, which far exceeded the current limit of $70 \mathrm{~A}$. The control current has fluctuations, which is related to the excessive gain. However, a small gain will cause the system to be unstable. The essential reason is that the MSL is a highly nonlinear system, and the PID or fuzzy PID are controllers based on linearization theory, which easily fails when applied to a nonlinear MLS. Additionally, the proposed controller is a nonlinear controller, which has not 
(a)

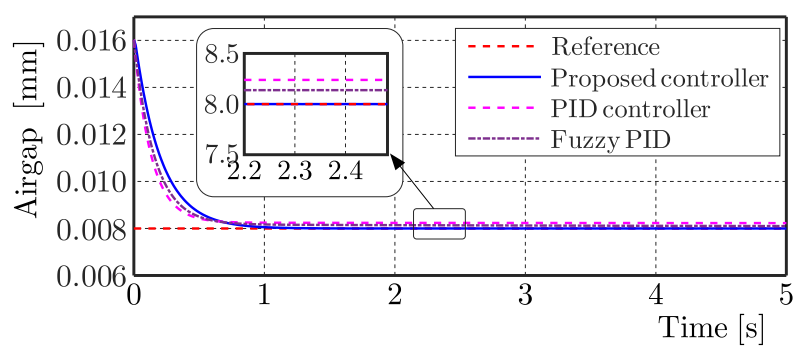

(b)

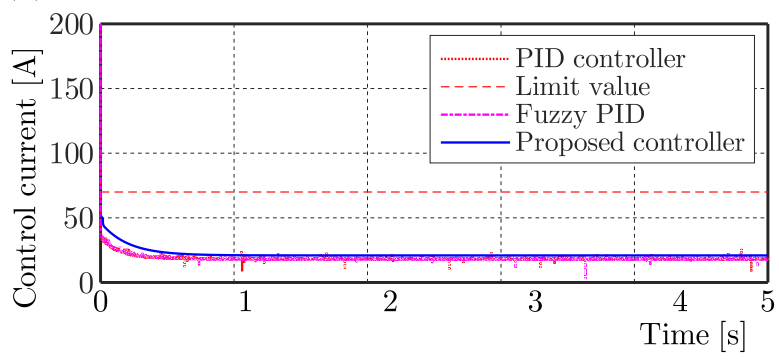

Fig. 4. (a) Airgap response in case 1, (b) control inputs in case 1

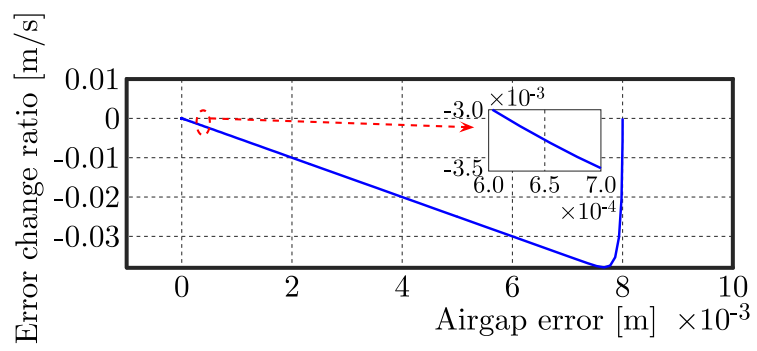

Fig. 5. Phase path with proposed controller in case 1

been linearized or simplified in the process of design, analysis and stability proof. The dynamic response of the airgap is better, and there is no overshoot and steady state error. The current maximum value is $55 \mathrm{~A}$, which is less than the system limit value of $70 \mathrm{~A}$. As shown in Fig. 5, the phase path of the proposed controller is smooth, which basically realizes removal of the oscillation of the control input.

Case 2. With the external disturbance

Discuss the dynamic performance of the PID, fuzzy PID and the proposed controller under the disturbance. The external disturbance is shown in Fig. 6.

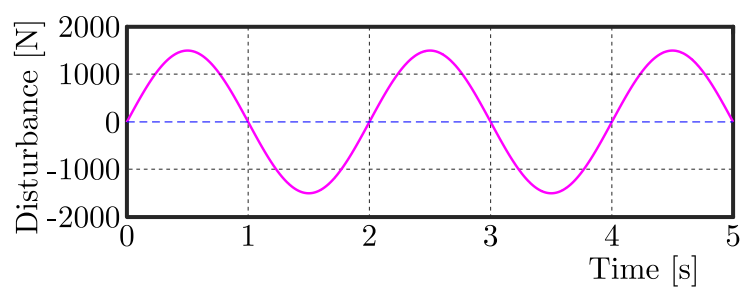

Fig. 6. Nonlinear external disturbance

The simulation results for the three controllers are shown in Fig. 7.

We can learn from Fig. 7 that the maximum error of the system with the PID controller is $0.4 \mathrm{~mm}$ and $0.2 \mathrm{~mm}$ for the fuzzy PID, and the control currents all exceed the physical limit of the actuator $70 \mathrm{~A}$. Under the action of the proposed controller, the maximum error of the system is $0.029 \mathrm{~mm}$. Moreover, the control current is smoother than the PID or fuzzy PID.

In order to further verify the increased performance over PID controllers, the amplitude in Fig. 6 is magnified three times with the frequency remain unchanged. The results of simulation for the airgap response are shown in Fig. 8.

We can learn from Fig. 8a that when the disturbance is increased three times, the PID controller cannot stabilize the system and the system is unstable. In the same situation, the proposed controller can stabilize the system near the target trajectory with the maximum error of $\pm 0.25 \mathrm{~mm}$. 
(a)

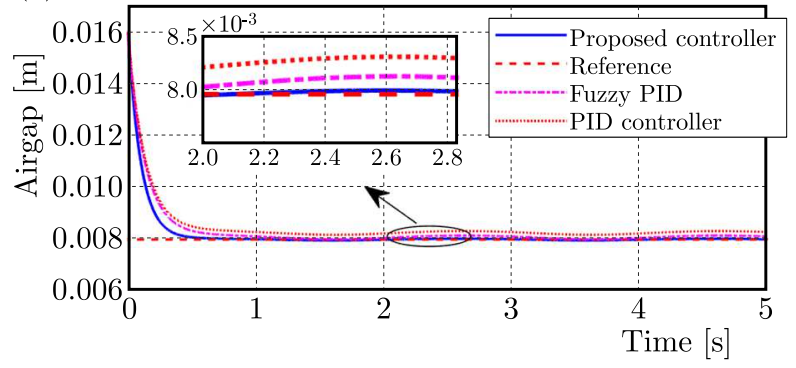

(b)

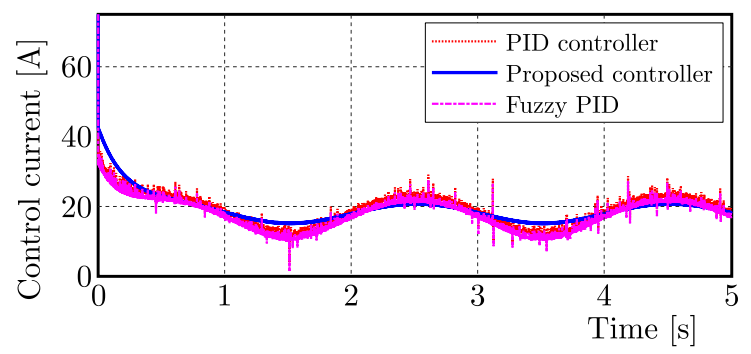

Fig. 7. (a) Airgap response in case 2. (b) control inputs in case 2

(a)

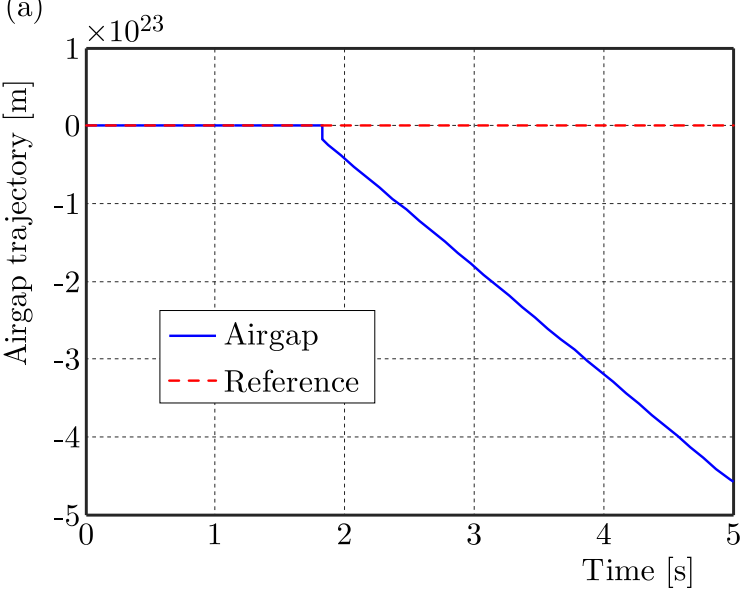

(b)

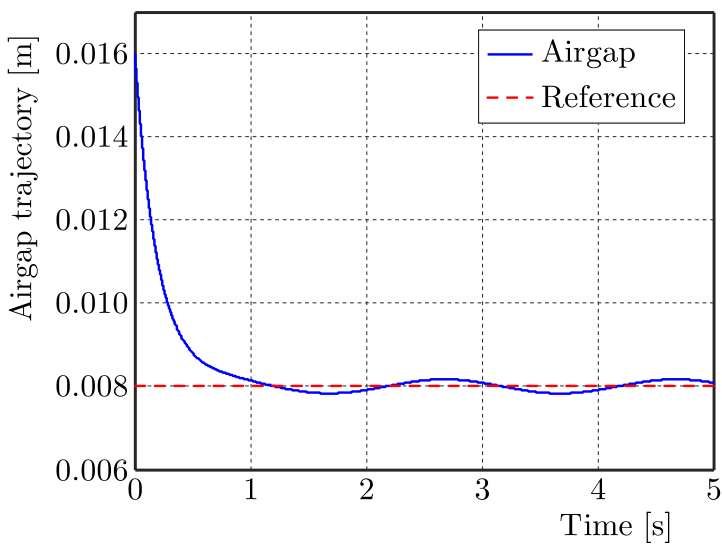

Fig. 8. Airgap with (a) PID and (b) proposed method under triple disturbance

Case 3. Parameter perturbations

The mass is increased up to 1.2 times of the nominal mass, and the simulation results are shown in Fig. 9.

(a)

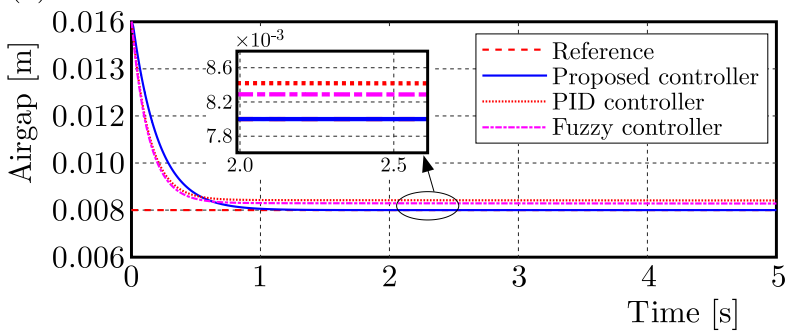

(b)

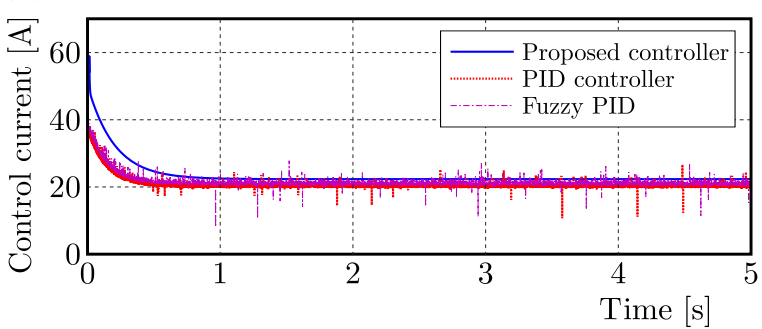

Fig. 9. (a) Airgap response in case 3, (b) control inputs in case 3

It can be seen from Fig. 9 that when the parameter is perturbed, there is a static error of $0.4 \mathrm{~mm}$ for the PID controller and $0.3 \mathrm{~mm}$ for the fuzzy PID controller. The control currents of these two controllers have some oscillations. The proposed controller is almost without a static error and overshoot during the levitation process. Moreover, the control current of the proposed controller is smooth and below the limit value of $70 \mathrm{~A}$.

In conclusion, the proposed controller designed by combining the backstepping method and the quasi-sliding mode control method with a variable boundary layer is an effective control method, which can satisfy the static and dynamic adjustment performance. The simulation 
results demonstrate that the proposed method has strong robustness to deal with the disturbance and parameters perturbation without oscillation.

\section{Experimental implementation and results}

After simulation tests, much effort has been put to perform experiments to further evaluate the performance of the proposed controller.

Since it is hard to build a full-scale maglev vehicle, a single magnetic levitation system of a maglev vehicle is established as illustrated in Fig. 10. It consists of an electromagnet, car-body load (can change by adding iron plates), secondary suspension and the dSPACE control system. The length of the electromagnet coil is $534 \mathrm{~mm}$. The suspension stiffness of the secondary suspension is $60 \mathrm{~N} / \mathrm{mm}$. The initial car-body load is $242 \mathrm{~kg}$. The airgap is measured by an eddy current sensor. The data recording frequency is $1 \mathrm{kHz}$.

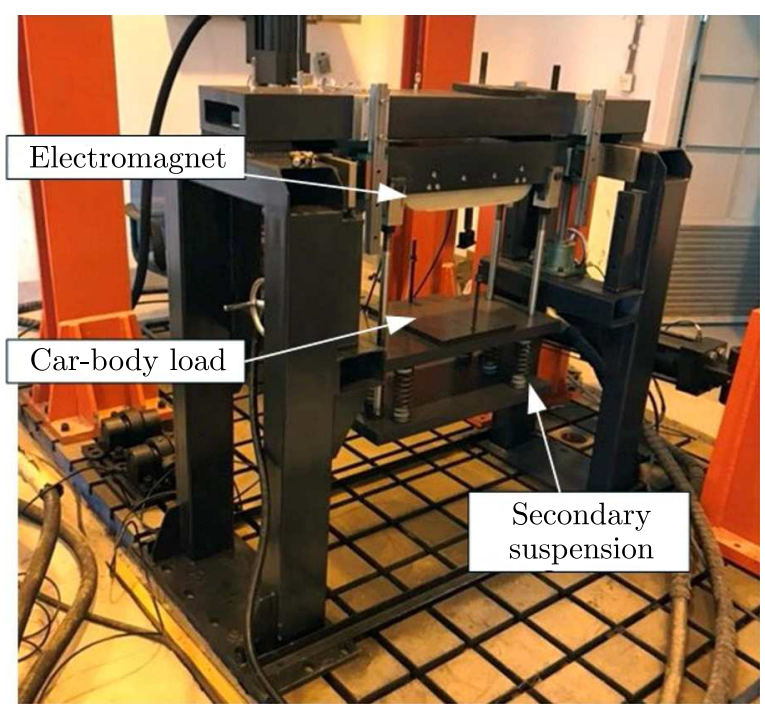

Fig. 10. Single magnetic levitation system with dSPACE

The experimental results of the proposed controller without parameters perturbation are provided in Fig. 11.

(a)

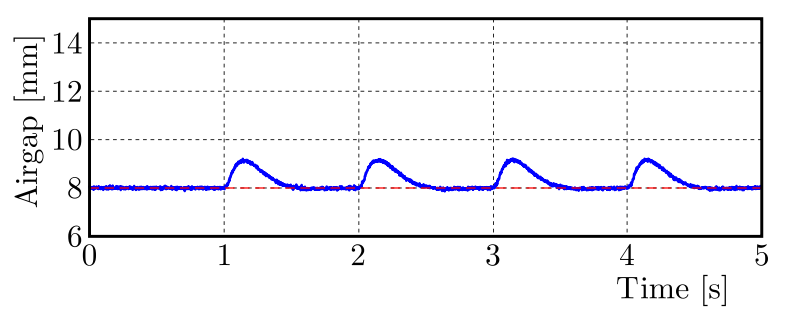

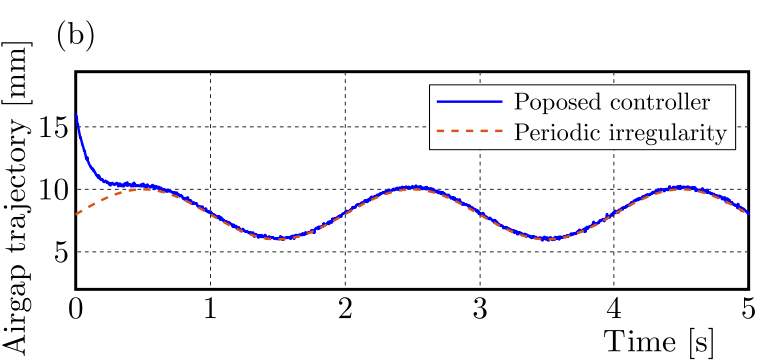

: (a) airgap, (b) current

It can be seen from Fig. 11 that the airgap response with the proposed controller can track the target trajectory, which is stable by about $1 \mathrm{~s}$, and the steady state error is 0 . The experimental current is relatively smooth, and the maximum current is $57 \mathrm{~A}$, which is less than the limit value of $70 \mathrm{~A}$.

In the experimental device, the car-body mass is increased by adding iron plates. Adjusting the mass to 1.3 times of the nominal mass, and at $1.5 \mathrm{~s}$ removing an iron plate (simulate passenger 
alighting) the mass is reduced to 1.1 times of the nominal mass. The dynamic response of the airgap is shown in Fig. 12.
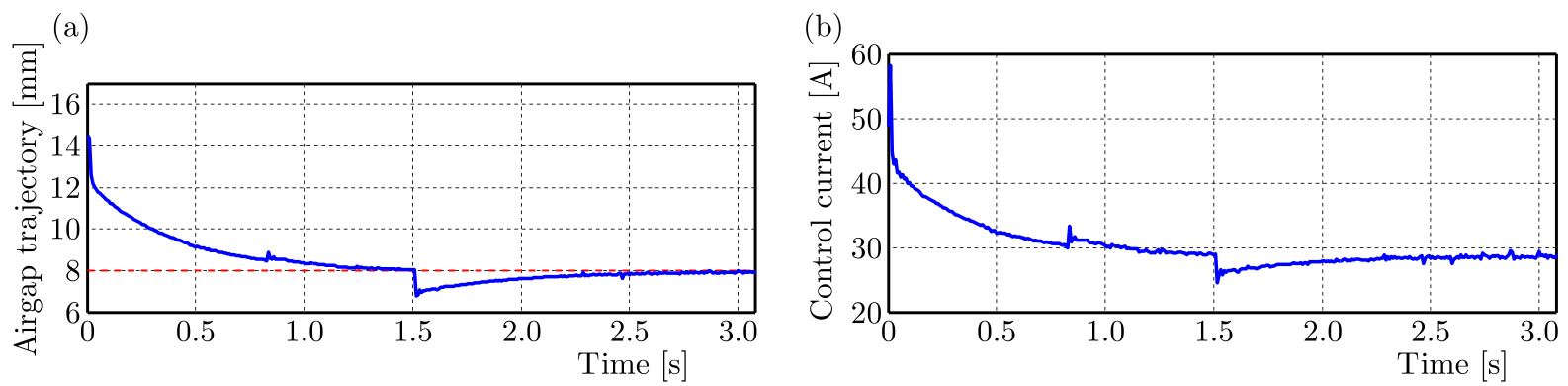

Fig. 12. Experimental results 2: (a) airgap, (b) current

It can be seen from Fig. 12a that when the mass becomes 1.3 times the nominal mass, the suspension can be durably stabilized, and it is stable within about $1.3 \mathrm{~s}$. The mass suddenly decreases and the airgap changes, but it can be stabilized again within $0.5 \mathrm{~s}$. The control current is smooth during the whole process, and there is no violent oscillation. However, the traditional PID leads to rail smashing and cannot be operated normally.

In practice, there are discontinuities in the magnetic track. We provided the experimental results of the airgap response by applying an impulse disturbance of $4 \mathrm{~mm}$ per second as shown in Fig. 13a. In addition, for high speeds at which maglev and railway vehicles travel, there are areas of the pass-band and stop-band of vibration due to wave propagation in the track (Shi et al., 2014; Chudzikiewicz et al., 2018; Huang et al., 2017; Sheng and Li, 2007). The experimental results of the MLS for tracking the periodic track irregularity is provided in Fig. 13b.
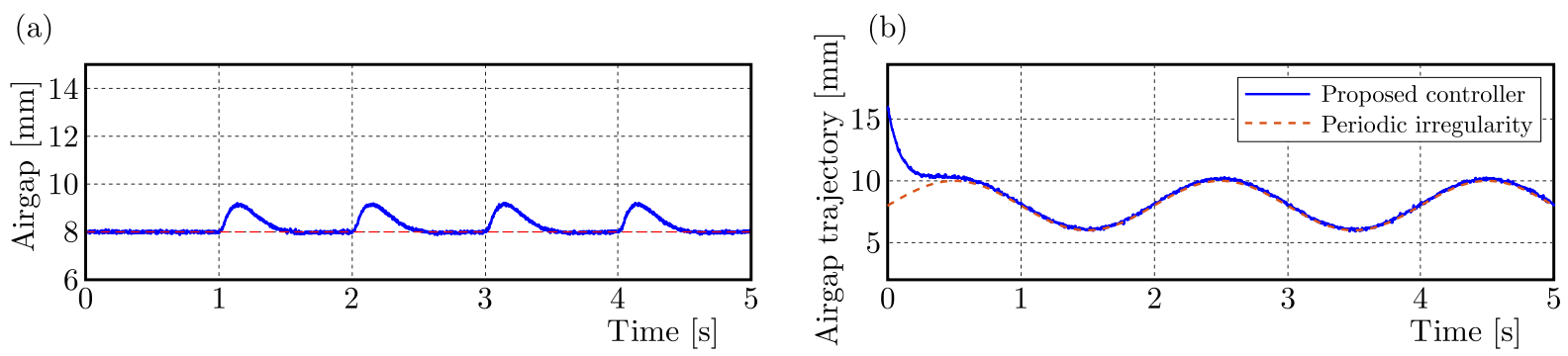

Fig. 13. Airgap with (a) impulse and (b) periodic disturbance

It can be seen from Fig. 13 that the proposed controller has the ability to deal with the impulse disturbance and periodic track irregularity.

In summary, the proposed controller is suitable for practical application in the MLS of the maglev train and can achieve excellent levitation control performances.

\section{Conclusion}

In this paper, a novel levitation controller based on the backstepping method and sliding mode control is presented to cope with the external disturbances and mass parameter changes of the MLS. However, in order to achieve accurate control, the maglev dynamic model needs to be added into the control law. However, nonlinear factors will cause large modeling errors. The Gaussian process method is utilized to overcome this problem. The proposed controller is designed by the backstepping method and the proof of stability is provided. The simulation results show that the proposed method can levitate the maglev train with more levitation accuracy, higher anti-disturbance and parameters uncertainty capacity. Finally, a single magnetic levitation system 
of the maglev vehicle is established. The experimental results demonstrate that the proposed controller is suitable for practical application in the MLS of the maglev train and has the ability to deal with an impulse disturbance and periodic track irregularity. However, for high speeds at which maglev and railway vehicles travel, there are areas of the pass-band and stop-band of vibration due to wave propagation in the track. Our future works will focus on the dynamic performance of the MLS of the maglev train that is in motion with various track irregularity excitations.

\section{Acknowledgment}

This work was supported in part by the National Natural Science Foundation of China under Grant 51905380 and Grant 52072269, in part by Shanghai Collaborative Innovation Center for Multi-Network and Multi-Mode Rail Transit, in part by the Shanghai Municipal Science and Technology Major Project (2021SHZDZX0100) and the Fundamental Research Funds for the Central Universities.

\section{References}

1. Boldea I., Tutelea L., Xu W., PucCi M., 2017, Linear electric machines, drives, and MAGLEVs: an overview, IEEE Transactions on Industrial Electronics, 65, 9, 7504-7515

2. Carvajal J., Chen G., Ogmen H., 2000, Fuzzy PID controller: Design, performance evaluation, and stability analysis, Information Sciences, 123, 3-4, 249-270

3. Chudzikiewicz A., Bogacz R., Kostrzewski M., Konowrocki R., 2018, Condition monitoring of railway track systems by using acceleration signals on wheelset axle-boxes, Transport, 33, $2,555-566$

4. Huang Y., Shi J., Wu Z.-W., Gao Y., Wang, D.-Z., 2017, Research on influence of line deviation for high-speed maglev transportation system, The 2017 World Congress on Advances in Structural Engineering and Mechanics (ASEM17), Ilsan, Seoul, Korea

5. Kusagawa S., Baba J., Shutoh K., Masada E., 2004, Multipurpose design optimization of EMS-type magnetically levitated vehicle based on genetic algorithm, IEEE Transactions on Applied Superconductivity, 14, 2, 1922-1925

6. Lee H.W., Kim K.C., LeE J., 2006, Review of maglev train technologies, IEEE Transactions on Magnetics, 42, 7, 1917-1925

7. Li J., Li J., Zhou D., Cui P., WAng L., Yu P., 2015, The active control of maglev stationary self-excited vibration with a virtual energy harvester, IEEE Transactions on Industrial Electronics, 62, 5, 2942-2951

8. LiU C., RonG G., 2015, SVM $\alpha$ order inverse system decoupling time-varying sliding mode control of double suspension systems of machining center, China Mechanical Engineering, 26, 5, 668-674

9. MacLeod C., Goodall R.M., 1996, Frequency shaping LQ control of maglev suspension systems for optimal performance with deterministic and stochastic inputs, IEE Proceedings - Control Theory and Applications, 143, 1, 25-30

10. Morales R., Feliu V., Sira-Ramirez H., 2011, Nonlinear control for magnetic levitation systems based on fast online algebraic identification of the input gain, IEEE Transactions on Control Systems Technology, 19, 4, 757-771

11. Quiñonero-Candela J., Rasmussen C.E., 2005, A unifying view of sparse approximate Gaussian process regression, Journal of Machine Learning Research, 6, 1939-1959

12. Sheng X., Li M.H., 2007, Propagation constants of railway tracks as a periodic structure, Journal of Sound and Vibration, 299, 4-5, 1114-1123

13. Shi J., FAng W.S., WANG Y.J., ZhaO, Y., 2014, Measurements and analysis of track irregularities on high speed maglev lines, Journal of Zhejiang University, Science A, 15, 6, 385-394 
14. Sinha P.K., Hadjisski L.M., Zhou F.B., Kutiyal R.S., 1993, Electromagnetic suspension: New results using neural networks, IEEE Transactions on Magnetics, 29, 6, 2971-2973

15. Sinha P.K., PEChev A.N., 1999, Model reference adaptive control of a maglev system with stable maximum descent criterion, Automatica, 35, 8, 1457-1465

16. Sun Y., Qiang H., Xu J., Lin G., 2020, Internet of things-based online condition monitor and improved adaptive fuzzy control for a medium-low-speed maglev train system, IEEE Transactions on Industrial Informatics, 16, 4, 2629-2639

17. Sun Y., Xu J., Qiang H., Lin G., 2019, Adaptive neural-fuzzy robust position control scheme for maglev train systems with experimental verification, IEEE Transactions on Industrial Electronics, 66, 11, 8589-8599

18. Sun Y., Xu J.Q., QIang H.Y., Wang W., Lin G., 2019, Hopf bifurcation analysis of maglev vehicle-guideway interaction vibration system and stability control based on fuzzy adaptive theory, Computers in Industry, 108, 197-209

19. Thornton R.D., 2009, Efficient and affordable maglev opportunities in the United States, Proceedings of the IEEE 97, 11, 1901-1921

20. WAI R.J., LEE J.D., 2008, Adaptive fuzzy-neural-network control for maglev transportation system, IEEE Transactions on Neural Networks, 19, 1, 54-70

21. Wu S.J., Wu C.T., Chang Y.C., 2008, Neural-fuzzy gap control for a current/voltage-controlled 1/4-vehicle MagLev system, IEEE Transactions on Intelligent Transportation Systems, 9, 1, 122-136

22. YAN L., 2008, Development and application of the maglev transportation system, IEEE Transactions on Applied Superconductivity, 18, 2, 92-99

23. Zhang G., Zhang J., Zhang H.-L., Meng Q.-T., Fan M., 2013, Calculation on magnetic force for permanent magnetic bearings by Monte Carlo method based on equivalent magnetic charge method, Bearing, 10, 1-4 